\title{
Nuclear Magnetic Resonance Study of ${ }^{23} \mathrm{Na}$ Nucleus in $\mathrm{NaBrO}_{3}$ Single Crystal
}

\author{
Tae Ho Yeom* \\ Department of Laser and Optical Information Engineering, Cheongju University, Cheongju 28503, Korea
}

(Received 26 September 2015, Received in final form 4 November 2015, Accepted 10 November 2015)

\begin{abstract}
The nuclear magnetic resonance of the ${ }^{23} \mathrm{Na}$ nucleus in a $\mathrm{NaBrO}_{3}$ single crystal was investigated at the temperature range of $200 \mathrm{~K} \sim 410 \mathrm{~K}$. The tendencies of temperature dependence of the nuclear quadrupole coupling for the two magnetically inequivalent $\mathrm{Na}$ (I) and $\mathrm{Na}$ (II) centers are found to be opposite to each other. The nuclear spin-lattice relaxation mechanism of ${ }^{23} \mathrm{Na}$ in the $\mathrm{NaBrO}_{3}$ crystal is investigated, and the result revealed that the Raman process is dominant in the temperature range investigated. The relaxation process of the ${ }^{23} \mathrm{Na}$ nuclear spins was well described by a single exponential function in time. The $\mathrm{T}_{1}$ values of the ${ }^{23} \mathrm{Na}$ nuclei in the $\mathrm{NaBrO}_{3}$ single crystal decreased with increasing temperature. The calculated activation energy for the ${ }^{23} \mathrm{Na}$ is $0.032 \pm 0.002 \mathrm{eV}$.
\end{abstract}

Keywords : magnetic resonance, $\mathrm{NaBrO}_{3}$ crystal, relaxation mechanism, activation energy

\section{Introduction}

Cubic $\mathrm{NaBrO}_{3}$ crystal is well known to exhibit many interesting optical properties including optical rotation and electro-optic effects. Stimulated Raman scattering (SRS) in $\mathrm{NaBrO}_{3}$ crystal to allow for the efficient generation of multiple Stokes and anti-Stokes emission was found by P. Franz et al. [1]. The $\mathrm{BrO}_{3}^{-}$groups occupy sites with a four-fold multiplicity and a symmetry of three. Compared to isomorphous and SRS-active $\mathrm{NaClO}_{3}$, $\mathrm{NaBrO}_{3}$ has been found to exhibit higher Vickers hardness and grinding hardness and a higher thermal stability. $\mathrm{NaBrO}_{3}$ is a nonlinear material for applications such as Raman shifters or Raman lasers in the visible and near-IR range [1].

Frequency shifting of pico- and nano-second laser pulses is of interest for ecology monitoring techniques and for the generation of specific multiple wavelength cascades covering wide spectral regions in the infrared and visible ranges. This motivated the realization of an all-solid-state Raman laser at the base of $\mathrm{NaBrO}_{3}$. With cubic $\mathrm{NaBrO}_{3}$, J. Findeisen et al. [2] realized for the first time an all solid-state Raman laser using a simple set-up. $\mathrm{NaBrO}_{3}$ crystal is a promising candidate for efficient Raman lasers in comparison to $\mathrm{Ba}\left(\mathrm{NO}_{3}\right)_{2}, \mathrm{KGd}\left(\mathrm{WO}_{4}\right)_{2}$, and $\mathrm{PbWO}_{4}$ [2].

(C)The Korean Magnetics Society. All rights reserved.

*Corresponding author: Tel: +82-43-229-8555

Fax: +82-43-229-8498, e-mail: thyeom@cju.ac.kr
The $\mathrm{NaBrO}_{3}$ crystal belongs to the cubic structure (acentric space group $\mathrm{P} 2{ }_{1} 3$ ) with the lattice constant $0.6555 \mathrm{~nm}$ [3]. The unit cell consists of four molecules (20 atoms). They form low trigonal pyramids. The structure is shown in Fig. 1. The bond between $\mathrm{Na}$ ion and $\mathrm{BrO}_{3}$ ion is generally ionic [4]. The $\mathrm{BrO}_{3}$ ion is a flat trigonal pyramid having oxygen atoms as its base. The binding between $\mathrm{O}-\mathrm{Br}$ is largely a covalent structure. From the symmetry of the flat trigonal pyramid of oxygens, the symmetrical axes of the gradient of the electric field at the nuclear spins of $\mathrm{Br}$ are the lines passing through the $\mathrm{Na}$ and $\mathrm{Br}$ nuclei. Fig. 1 shows this axis of symmetry.

For the Na-Br distances in $\mathrm{NaBrO}_{3}$, the ionic radius of $\mathrm{Na}$ ions is $0.095 \mathrm{~nm}$ and that of $\mathrm{Br}$ ion is $0.195 \mathrm{~nm}$, while their sum is $0.290 \mathrm{~nm}$ which is shorter than the nearest distance of Na-Br of $0.342 \mathrm{~nm}$. This means that the binding between $\mathrm{Na}$ and $\mathrm{Br}$ is partly covalent and the nuclear spin levels of $\mathrm{Na}$ has quadrupole splittings, while the symmetry axis of the field gradient at the $\mathrm{Na}$ nucleus is the same as that of $\mathrm{Br}[5]$.

Several nuclear quadrupole resonances of $\mathrm{NaBrO}_{3}$ crystals [6-9] have been studied for many years. Nuclear magnetic resonance (NMR) is a good technique for the investigation of microscopic information on the materials. The spin-lattice relaxation rate of the nuclei in the host crystal reflects the dynamics of the crystal, such as the nucleus-phonon interaction, and indicates the ease at which the excited state energy of the nuclear system is transferred into the lattice. In this paper, we measured the 

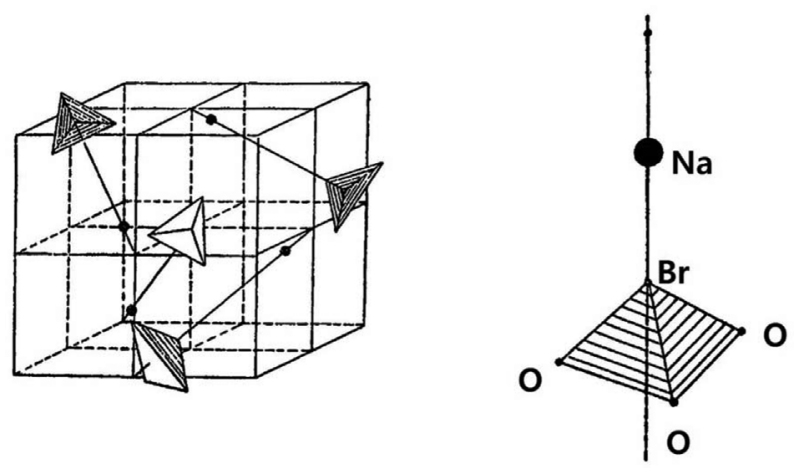

Fig. 1. Crystal structure of $\mathrm{NaBrO}_{3}$ in the unit cell. The vertical line is the symmetric axis of the electric field gradient at the $\mathrm{Na}$ and $\mathrm{Br}$ sites.

NMR spectra and spin-lattice relaxation time of a ${ }^{23} \mathrm{Na}$ nucleus by using a pulse NMR spectrometer to obtain detailed information about the dynamics of a $\mathrm{NaBrO}_{3}$ crystal. This paper documents these new investigations of NMR for the $\mathrm{NaBrO}_{3}$ crystal.

\section{Experiments}

The spin-lattice relaxation times $T_{1}$ of the ${ }^{23} \mathrm{Na}$ in $\mathrm{NaBrO}_{3}$ single crystals were measured using the Bruker $200 \mathrm{MHz}$ FT NMR spectrometer at the Korea Basic Science Institute Seoul Western Center. The static magnetic field was $4.7 \mathrm{~T}$ and the central radio frequency was set to $\omega_{o} / 2 \pi=52.9015 \mathrm{MHz}$ for the ${ }^{23} \mathrm{Na}$ nucleus. The spinlattice relaxation times were measured using the saturation recovery pulse sequence, sat- $t-\pi / 2$-acq, for the ${ }^{23} \mathrm{Na}$ nucleus. The width of the $\pi / 2$ pulse was $1.8 \mu$ s for the ${ }^{23} \mathrm{Na}$ nucleus. The temperature-dependent NMR measurements were obtained at the temperature range of 200-410 $\mathrm{K}$. The samples were maintained at constant temperatures by controlling the nitrogen gas flow and heater current.

The ${ }^{23} \mathrm{Na}$ NMR spectrum in $\mathrm{NaBrO}_{3}$ single crystal at room temperature is shown in Fig. 2 when the magnetic field B was applied along the crystallographic c-axis. This spectrum is obtained by Fourier transform of the free induction decay for the ${ }^{23} \mathrm{Na}$ NMR. Three resonance lines are expected because of the quadrupole interaction of the ${ }^{23} \mathrm{Na}(I=3 / 2,100 \%)$ nuclei with the electric field gradient (EFG) under a strong magnetic field. However, six resonance lines are obtained in all temperature ranges when magnetic field $\mathrm{B}$ is parallel to the crystallographic c-axis. The line width of the central line for the ${ }^{23} \mathrm{Na}$ nucleus at room temperature is about $3 \mathrm{kHz}$. However, while the central transition remains constant, the interval between the central and satellite lines is found to change as the temperature increases.

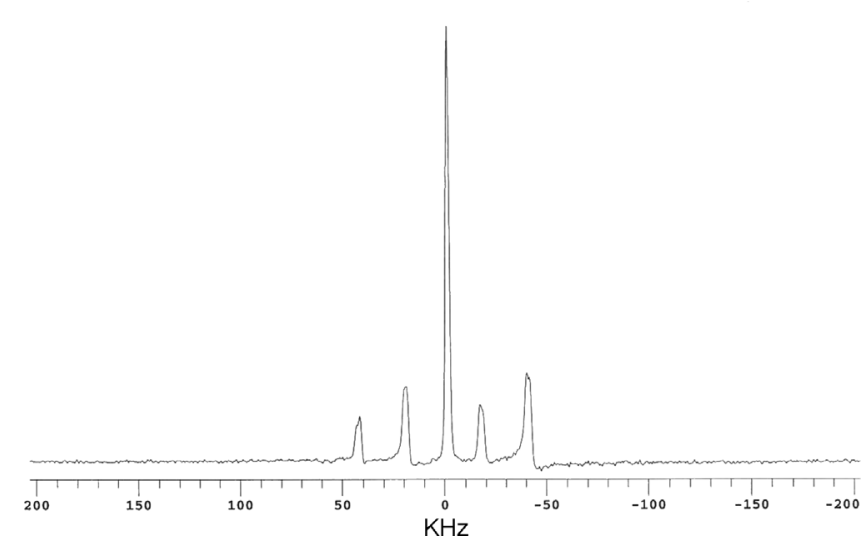

Fig. 2. Typical nuclear magnetic resonance spectrum of ${ }^{23} \mathrm{Na}$ nucleus at $300 \mathrm{~K}$ operating at $\omega_{o} / 2 \pi=52.9015 \mathrm{MHz}$. The inner satellite line within $\pm 30 \mathrm{kHz}$ is denoted as $\mathrm{Na}$ (I) center, whereas the outer side $\pm 30 \mathrm{kHz}$ is denoted as $\mathrm{Na}$ (II) center.

\section{Analysis and Discussion}

Each unit cell has four $\mathrm{Na}$ ions. In the $\mathrm{NaBrO}_{3}$ crystal, six NMR lines of Na nucleus were obtained at all temperature ranges investigated. One of the typical NMR spectra of ${ }^{23} \mathrm{Na}$ along the c-axis at room temperature is shown in Fig. 2. The four Na nuclei in the unit cell give two sets of NMR signals (six resonance signals) for the ${ }^{23} \mathrm{Na}$ nuclei in the $\mathrm{NaBrO}_{3}$ crystal across the temperature range investigated. Two Na nuclei in the unit cell give a $\mathrm{Na}(\mathrm{I})$ NMR center and the other two Na nuclei in the unit cell give a $\mathrm{Na}(\mathrm{II}) \mathrm{NMR}$ center. The inner satellite lines within $\pm 30 \mathrm{kHz}$ are denoted as a $\mathrm{Na}(\mathrm{I})$ center, whereas those outside $\pm 30 \mathrm{kHz}$ are denoted as a $\mathrm{Na}$ (II) center. Two sets of NMR signals originated from the $\mathrm{Na}$ (I) and $\mathrm{Na}$ (II) centers. The central lines of the $\mathrm{Na}(\mathrm{I})$ and $\mathrm{Na}$ (II) centers merge into one resonance line. We found that two sets of NMR signals originated from the crystal symmetry, rather than from the twin structure of the $\mathrm{NaBrO}_{3}$ crystal. The central transition $(|1 / 2>\leftrightarrow|-1 / 2>)$ was not shifted by the quadrupole interaction, but the satellite transitions $([3 / 2>$ $\leftrightarrow \mid 1 / 2>)$ and $(|-1 / 2>\leftrightarrow|-3 / 2>)$ were shifted. The line width for ${ }^{23} \mathrm{Na}$ at room temperature is about $3 \mathrm{kHz}$ and slightly decreases with increasing temperature.

The nuclear quadrupole splitting of the $\mathrm{Na}(\mathrm{I})$ center is found to increase with increasing temperature, whereas that of the $\mathrm{Na}(\mathrm{II})$ center is found to decrease with increasing temperature. The trend of the $\mathrm{Na}(\mathrm{I})$ center is opposite that for the $\mathrm{Na}$ (II) center, as shown in Fig. 3. The temperature dependence of the quadrupole splitting is usually analyzed in terms of the Bayer theory, taking into account only the rigid-body vibration of the molecule [10-12]. The obtained temperature dependence of the quadrupole 


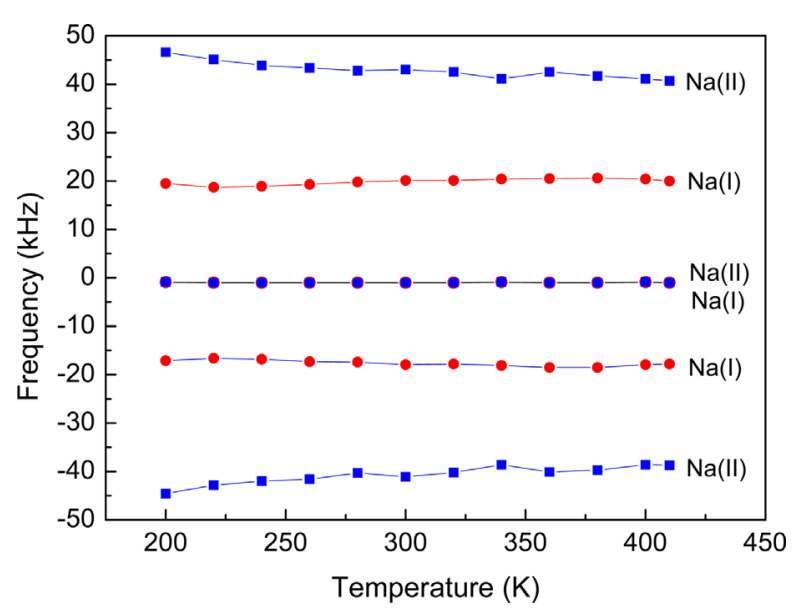

Fig. 3. (Color online) Zeeman splitting of NMR spectrum for ${ }^{23} \mathrm{Na}$ in $\mathrm{NaBrO}_{3}$ single crystal. The zero point is 52.9015 $\mathrm{MHz}$.

coupling constant (QCC) of the $\mathrm{Na}$ (II) center can be explained with a single torsional mode for $\mathrm{Na}(\mathrm{II})$. However, the Bayer theory cannot explain the temperature dependence on QCC of the $\mathrm{Na}(\mathrm{I})$ center shown in Fig. 3, because the theory will always predict a decreasing coupling constant with increasing temperature since the amplitude of the rigid body vibration increases with temperature.

The spin-lattice relaxation times $T_{1}$ for ${ }^{23} \mathrm{Na}$ in $\mathrm{NaBrO}_{3}$ single crystals were measured as a function of temperature. The saturation recovery traces of the magnetization were measured at a temperature range of $200-410 \mathrm{~K}$ for the ${ }^{23} \mathrm{Na}$ nucleus. The measured magnetization recoveries $[M(\infty)-M(t)] / 2 M(\infty)$ for the ${ }^{23} \mathrm{Na}(I=3 / 2)$ nucleus were found to satisfactorily fit a single exponential function with $[13,14]$.

$$
[M(\infty)-M(t)] / 2 M(\infty)=\exp \left(-t / T_{1}\right),
$$

where $M(\infty)$ and $M(t)$ are the thermal equilibrium and time-dependent magnetizations, respectively, $t$ is the time between the first and second pulses, and $T_{1}$ is the spinlattice relaxation time.

The relaxation processes of the magnetic moment of the ${ }^{23} \mathrm{Na}$ nucleus as a function of delay time $\mathrm{t}$ are shown in Fig. 3 at temperatures of $220 \mathrm{~K}, 300 \mathrm{~K}$, and $400 \mathrm{~K}$. The relaxation of the ${ }^{23} \mathrm{Na}$ nuclear spins is found at a single exponential in time when all the resonance lines are uniformly excited. The $T_{1}$ values obtained by exciting only one line and by exciting all the lines are theoretically identical. Experimental data denoted by symbols fit well to Eq. (1) for each temperature.

The interaction of the nuclear quadrupole moment with the lattice vibration is a very important relaxation mechanism for the nuclear spin $I \geq 1$ in many crystals.

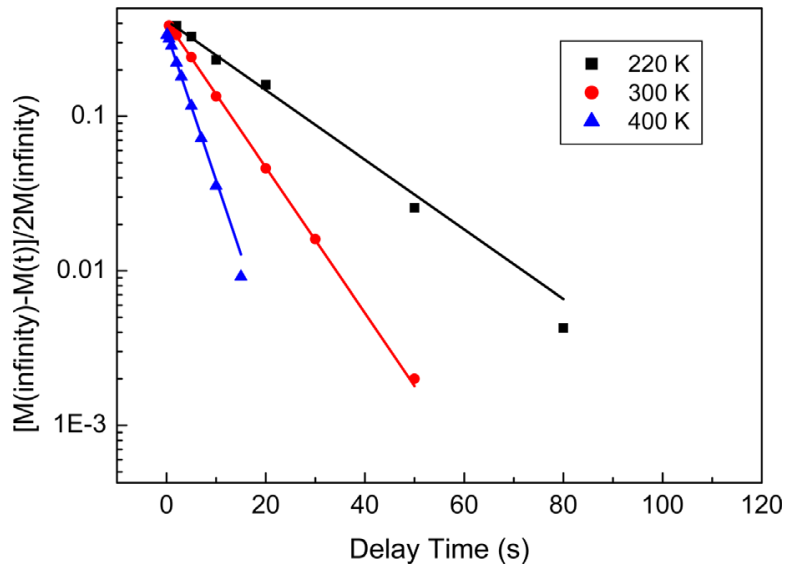

Fig. 4. (Color online) Magnetization recovery traces of ${ }^{23} \mathrm{Na}$ in $\mathrm{NaBrO}_{3}$ crystal as a function of delay time at several different temperatures. The solid lines are fitted using Eq. (1).

One of the major contributions to the spin-lattice relaxation for the nuclear spin $I \geq 1$ could be the quadrupolar interaction of the electric quadrupole moment of the nucleus with the lattice vibrations. The coupling can generally be written with a spin-lattice Hamiltonian [15]. At a temperature considerably below the melting point of the crystal, we can reasonably assume that the thermal stress should be small and only the first few terms are important. The first order term represents the absorption or emission of a single phonon (direct process). The next second order term indicates the emission or absorption of two phonons, or the absorption of one phonon followed by the emission of another phonon (Raman process). In the direct process, the spin-lattice relaxation rate $1 / T_{1}$ is proportional to the square of the Larmor frequency for the nucleus, $\omega_{0}{ }^{2}$, and to the absolute temperature $T$. On the other hand, the Raman process provides a relaxation rate proportional to the square of the temperature at the high temperature limit $[16,17]$. The Orbach process is another two-phonon process, but it provides an exponential behavior with temperature at the relaxation rate [15].

The temperature dependence of the nuclear spin-lattice relaxation rate $1 / T_{1}$ for the ${ }^{23} \mathrm{Na}$ nucleus in the $\mathrm{NaBrO}_{3}$ crystal was obtained and is shown in Fig. 5. The relaxation time of the ${ }^{23} \mathrm{Na}$ nuclei does not undergo any abrupt changes within the experimental temperature range of 200-410 K, which indicates that no phase transitions occur within this temperature range. The $1 / T_{1}$ values of ${ }^{23} \mathrm{Na}$ were found to increase with an increase in the temperature. This is reasonable because the lattice vibration is more effective when the crystal temperature is increased, and the relaxation time is then shorter with increasing temperature.

The $T_{1}$ values for ${ }^{23} \mathrm{Na}$ in the $\mathrm{NaBrO}_{3}$ crystal can be 


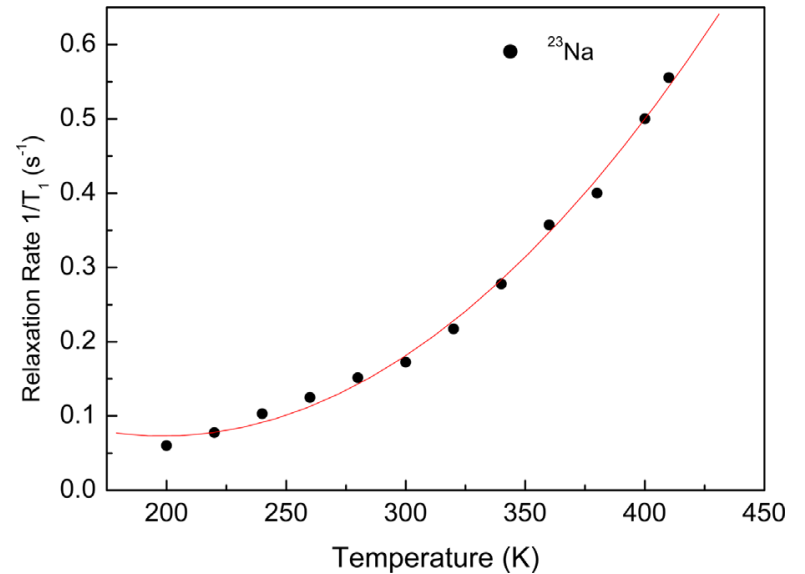

Fig. 5. (Color online) Temperature dependence of spin-lattice relaxation rate $1 / T_{1}$ for ${ }^{23} \mathrm{Na}$ in $\mathrm{NaBrO}_{3}$ crystal. The solid line is fitted by assuming Raman processes.

explained in terms of a relaxation mechanism in which lattice vibrations are coupled to the nuclear electric quadrupole moments. The relaxation rate is proportional to the square of the temperature for the nuclei in the investigated temperature range (solid line in Fig. 5). Therefore, the temperature dependence of the ${ }^{23} \mathrm{Na}$ relaxation rate in the $\mathrm{NaBrO}_{3}$ crystal is in accordance with Raman processes. The Raman process is more effective than the direct process for nuclear quadrupole relaxation in our experiment. Namely, the dominant relaxation mechanism for the nuclei is the coupling of these moments to the thermal fluctuations of the local electric field gradients via the Raman spin-phonon process. Since our measurement was taken with a single crystal sample with no

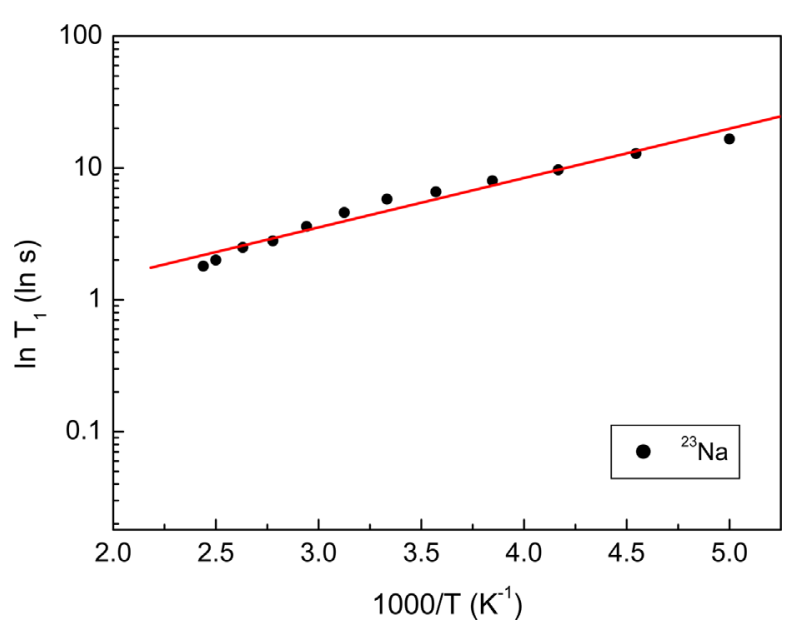

Fig. 6. (Color online) Semi log plot of spin-lattice relaxation time vs. the reciprocal temperature in the entire temperature region. The slope of the solid line represents the activation energy. appreciable impurities, the present result represents a more reliable feature of the intrinsic behavior of the relaxation process in $\mathrm{NaBrO}_{3}$.

The activation energy of the ${ }^{23} \mathrm{Na}$ nuclei in $\mathrm{NaBrO}_{3}$ single crystals was determined using the equation $T_{1}=$ $A_{o} \exp \left(E_{a} / k_{B} T\right)$, where $E_{a}$ and $A_{o}$ are the activation energy and the pre-exponential factor, respectively, while $k_{B}$ and $T$ are the Boltzmann constant and the temperature, respectively. The plot of the natural logarithm of $T_{1}$ as a function of inverse temperature is hence linear, with a slope that is directly related to the activation energy. From the gradient of the graph in Fig. 6, we obtained the activation energy of $0.032 \pm 0.002 \mathrm{eV}$, which is the energy required to activate the nucleus and to allow it to participate in the spin-lattice relaxation mechanism of the of ${ }^{23} \mathrm{Na}$ nucleus in the $\mathrm{NaBrO}_{3}$ single crystal.

\section{Conclusions}

The NMR of the ${ }^{23} \mathrm{Na}$ nucleus in the $\mathrm{NaBrO}_{3}$ single crystal was investigated by employing a FT-NMR spectrometer in the temperature range of $200 \mathrm{~K}-410 \mathrm{~K}$. Two sets of ${ }^{23} \mathrm{Na}$ spectra are obtained when $\mathrm{B} / / \mathrm{c}$-axis. These two sets of spectra are found to originate from the two magnetically inequivalent $\mathrm{Na}(\mathrm{I})$ and $\mathrm{Na}(\mathrm{II})$ sites in a $\mathrm{NaBrO}_{3}$ single crystal. The nuclear quadrupole coupling constant of the $\mathrm{Na}(\mathrm{I})$ center actually increases with increasing temperature, whereas that of the $\mathrm{Na}$ (II) center decreases with increasing temperature. The tendencies of the temperature dependence of the nuclear quadrupole coupling for the two magnetically inequivalent $\mathrm{Na}(\mathrm{I})$ and $\mathrm{Na}$ (II) centers are found to be opposite each other. The line width of the central line for the ${ }^{23} \mathrm{Na}$ nucleus at room temperature is about $3 \mathrm{kHz}$.

From the ${ }^{23} \mathrm{Na}$ NMR study, the relaxation mechanism of ${ }^{23} \mathrm{Na}$ was investigated by determining the spin-lattice relaxation time, $T_{1}$, of the ${ }^{23} \mathrm{Na}$ in the $\mathrm{NaBrO}_{3}$ crystal. The magnetization recoveries for the ${ }^{23} \mathrm{Na}$ nuclear spins were found to satisfactorily fit a single exponential function in time when all the resonance lines are uniformly excited. The spin-lattice relaxation time $T_{1}$ values of the ${ }^{23} \mathrm{Na}$ nuclei in the $\mathrm{NaBrO}_{3}$ single crystal continuously decreased with increasing temperature. This means that phase transitions do not occur within the experimental temperature range. The dominant relaxation mechanism in the investigated temperature range can be inferred as the Raman process because the spin-lattice relaxation rate 1/ $T_{1}$ of the ${ }^{23} \mathrm{Na}$ nucleus in the $\mathrm{NaBrO}_{3}$ crystal is proportional to the square of the temperature $\left(T^{2}\right)$. Namely, the lattice vibrations are coupled to the nuclear electric quadrupole moments; the dominant relaxation mechanism 
is the coupling of these moments to the thermal fluctuations of the local electric field gradients via the Raman spin-phonon process. The activation energy calculated for the ${ }^{23} \mathrm{Na}$ nucleus in the $\mathrm{NaBrO}_{3}$ single crystal is $0.032 \pm$ $0.002 \mathrm{eV}$.

\section{References}

[1] P. Franz, P. Egger, J. Hulliger, J. Findeisen, A. A. Kaminskii, and H. J. Eichler, Phys. Stat. Sol. (b) 210, R7 (1998).

[2] J. Findeisen, J. Hulliger, A. A. Kaminskii, H. J. Eichler, R. Macdonald, P. Franz, and P. Peuser, Phys. Stat. Sol. (a) 172, R5 (1999).

[3] R. W. G. Wyckoff, Crystal Structures, Inter-science Publishers, Inc., New York (1953).

[4] L. Pauling, The Nature of the Chemical Bond, Oxford Univ. Press, London (1952).

[5] K. Kano, J. Phys. Soc. Jan. 13, 975 (1958).

[6] R. F. Tipsword, J. T. Allender, E. A. Stahl, and C. D.
Williams, J. Chem. Phys. 49, 2464 (1968).

[7] C. J. Whidden, C. D. Williams, and R. F. Tipsword, J. Chem. Phys. 50, 507 (1969).

[8] D. D. Early, R. F. Tipsword, and C. D. Williams, J. Chem. Phys. 55, 460 (1971).

[9] E. A. Stahl and R. F. Tipsword, Magn. Resonance in Chemistry 28, 95 (1990).

[10] H. Bayer, Z. Physik 130, 227 (1951).

[11] T. C. Wang, Phys. Rev. 99, 566 (1955).

[12] T. P. Das and E. L. Han, Nuclear Quadrupole Resonance Spectroscopy, Academic Press, New York (1958) Chap. 1.

[13] W. W. Simmons, W. J. O'Sullivan, and W. A. Robinson, Phys. Rev. 127, 1168 (1962).

[14] W. E. Blumberg, Phys. Rev. 119, 79 (1960).

[15] A. Abragam, The Principles of Nuclear magnetism, Oxford Univ. Press, Oxford (1961) Chaps. I and IX.

[16] R. L. Mieher, Phys. Rev. 125, 1537 (1962).

[17] J. Van Kranendonk, Theory of quadrupolar nuclear spinlattice relaxation, Physica 20, 781 (1954). 